Check for updates

Cite this: RSC Adv., 2017, 7, 31033

\title{
Temperature-responsive methacrylamide polyampholytes $\uparrow$
}

\author{
Lucy G. Weaver, (D) a Regine Stockmann, ${ }^{a}$ San H. Thang ${ }^{\text {bc }}$ and Almar Postma (D) *b
}

A series of methacrylamide-based polyampholytes were synthesised for the first time and their temperature responsive properties investigated. $N$-[3-(Dimethylamino)propyl]methacrylamide (DMAPMAm) and methacrylic acid (MAA) were copolymerised in equimolar amounts along with $\mathrm{N}$-(tert-butyl) methacrylamide (tBMAm) via RAFT polymerisation, forming a series of polymers with varying tBMAm content. A variety of solvents were assessed to determine the optimal conditions for synthesis. The solution properties of the resultant polymers were then determined via turbidimetry and NMR. Results from this study show that tBMAm was an effective modulator of $T_{\mathrm{CP}}$ events when it was present in levels of $16 \%$ or more of the total monomers in the polymer chain. Below this threshold, polymers displayed properties akin to traditional polyampholytes due to the antipolyelectrolyte effect. Above this level however, the polymers displayed properties analogous to other temperature-responsive polymer systems. Interestingly changes in both $\mathrm{pH}$ and addition of salt led to an increase in the polymer cloud points, and in some cases loss of thermoresponsivity entirely, despite the high level of anionic and cationic charges present in the side chains.

Received 27th April 2017

Accepted 6th June 2017

DOI: 10.1039/c7ra04723a

rsc.li/rsc-advances the minimum of the bimodal of the polymer phase diagram. ${ }^{19}$ At different polymer concentrations this transition is visually observed as a clouding of the otherwise transparent polymerwater solution at a particular temperature (cloud point temperature; $\left.T_{\mathrm{CP}}\right) \cdot{ }^{20}$ As cloud temperatures are specific to each individual polymer, they can be modulated to higher or lower temperatures simply by changing the polymer composition to be more hydrophilic or hydrophobic, respectively. ${ }^{18}$ The cloud point of a polymer in solution can also be modified by including charged moieties that are inherently responsive to changes in $\mathrm{pH}$ or salt concentrations..$^{21,22}$

Our research group is focussing on the novel separation of analytes from aqueous food or food by-product streams. ${ }^{23,24}$ Therefore, we are developing new stimuli-responsive polymer systems with specifically engineered properties, such as mechanical strength and variant $\mathrm{pH}$ stability. Under challenging conditions, such as solution $\mathrm{pH}$ extremes, many of the common acrylate and methacrylate thermoresponsive copolymers with ester-linked side chains are immediately rendered noncompatible due to the propensity of the ester-linked side chain to be hydrolysed. ${ }^{25-29} \mathrm{~N}$-Substituted (meth)acrylamide copolymers, however, present a viable alternative to their ester-linked counterparts, with their stability demonstrated. ${ }^{30}$ For our applications, polymers must also have a high level of anionic and cationic groups along the polymer chain to enable interaction with specific analytes. Polymers with these properties in a single chain are usually referred to as polyampholytes, and typically possess zwitterionic functionality. These polymers are distinct in both function and structure from polyelectrolytes, which only 
contain a single type of charged group (anionic or cationic) in the polymer chain. Charged groups in polyampholytes are usually distributed on separate side chain units, as distinct from polybetaines where the charges are combined on a single side chain unit. Whilst both polyampholytes and polybetaines are polyzwitterions, their difference in structure can lead to markedly different interactions of the polymers with target analytes. ${ }^{31-33}$

The solution properties of polyampholytes are governed by coulombic interactions between charged moieties in the polymer chains and they can display both polyelectrolyte and antipolyelectrolyte behaviour depending on the solution properties. ${ }^{31}$ The antipolyelectrolyte effect (APE) occurs when a polyampholyte is in the presence of low or no added salt and self-polymer interactions cause the polymer to be in a globule state, with extension to a random coil only achieved via the addition of a low molecular weight salt. ${ }^{31}$ In contrast to this, polymers that display the polyelectrolyte effect exhibit a random coil state and high solubility in pure water, with the cloud point of solutions usually decreasing as a the salt concentration is increased - typical of classic thermoresponsive polymers. ${ }^{19}$ Therefore, temperature-responsive polyampholytes present a fascinating dichotomy in solution properties that can be controlled by changes in $\mathrm{pH}$ and salt concentrations and copolymer composition. These properties will herein be investigated via novel polyampholyte copolymers containing weakly acidic and basic species in the one polymer chain. ${ }^{31}$

\section{Experimental}

\section{Materials}

$N$-[3-(Dimethylamino)propyl]methacrylamide (DMAPMAm), methacrylic acid (MAA) and 1,3,5-trioxane were purchased from Sigma-Aldrich. DMAPMAm and MAA were purified by running the monomer through a short column packed with hydroquinone monomethyl ether inhibitor removers (Sigma-Aldrich). $\mathrm{N}$ (tert-Butyl)methacrylamide (tBMAm) was purchased from TCI Chemicals and was used as received. 4-(((2-Carboxyethyl)thio) carbonothioyl)thio)-4-cyanopentanoic acid (CETCPA) was obtained from Boron Molecular (BM1433) and was used as received. 4,4'-Azobis(4-cyanovaleric acid) (ACVA) was obtained from Sigma-Aldrich, and was recrystallised from methanol prior to use. All analytical grade solvents were purchased from SigmaAldrich or Merck and were used as received. NMR solvents were purchased from Cambridge Isotope Laboratories and were used as received.

\section{Instrumentation}

Gel Permeation Chromatography (GPC) for all polymers was carried out on a Waters Alliance system equipped with an Alliance 2695 Separations Module (integrated quaternary solvent delivery, solvent degasser and autosampler system), a Waters column heater module, a Waters 2414 RDI refractive index detector, a Waters PDA 2996 photodiode array detector $(210-400 \mathrm{~nm}$ at 1.2 $\mathrm{nm})$ and $2 \times$ Agilent PL-Aquagel columns (PL-Aquagel Mixed $\mathrm{H}(8$ $\mu \mathrm{m})$ ), each $300 \mathrm{~mm} \times 7.8 \mathrm{~mm}^{2}$, providing an effective molar mass range of 6000 to $6 \times 10^{7}$. The mobile phase was prepared with
Milli-Q grade water with $200 \mathrm{mM}$ sodium nitrate, $10 \mathrm{mM}$ sodium phosphate and $200 \mathrm{ppm}$ sodium azide at $\mathrm{pH} 8$ and was filtered through a $0.45 \mu \mathrm{m}$ membrane filter. The eluent had a flow rate of 1 $\mathrm{mL} \min ^{-1}$ at $30^{\circ} \mathrm{C}$. Number average $\left(M_{\mathrm{n}}\right)$ and weight average $\left(M_{\mathrm{w}}\right)$ molar masses were evaluated using Waters Empower-3 software. The GPC columns were calibrated with low dispersity polyethylene glycol/oxide (PEG/PEO) standards (Polymer Laboratories) ranging from 233 to $1016921 \mathrm{~g} \mathrm{~mol}^{-1}$, and molar masses are reported as PEG/PEO equivalents. A $3^{\text {rd }}$-order polynomial was used to fit the $\log M_{\mathrm{p}} v s$. time calibration curve, which was near linear across the molar mass ranges. Polymer samples were freeze dried using a CHRIST Alpha 1-2 LDplus freeze dryer with Leybold Rotary Vane Vacuum Pump (Trivac D4B) attached and with the chamber cooled to $-57^{\circ} \mathrm{C}$. Proton nuclear magnetic resonance $\left({ }^{1} \mathrm{H}\right.$ NMR) spectra were recorded on a Bruker BioSpin $400 \mathrm{MHz}$ NMR spectrometer (Billerica, MA) at $298 \mathrm{~K}$ and using a $10 \mathrm{~s}$ delay between acquisitions. This was found to be a sufficient relaxation time to allow accurate analysis of vinyl peaks for monomer consumption and monomer conversion calculations for these polymers. Data was analysed using TopSpin software. Chemical shifts $\left(\delta_{\mathrm{H}}\right)$ are reported in parts per million (ppm) and referenced to residual solvent signal (DMSO: $\delta 2.5 \mathrm{ppm}$ ) or internal standard TSP- $d_{4}$ : $\delta 0.0 \mathrm{ppm}$ in $\mathrm{D}_{2} \mathrm{O}$. In situ temperature ramp NMR experiments were also performed on a Bruker BioSpin $400 \mathrm{MHz}$ NMR spectrometer. Samples in $\mathrm{D}_{2} \mathrm{O}$ (no TSP- $d_{4}$ ) were loaded into the spectrometer at $298 \mathrm{~K}$ and heated to $348 \mathrm{~K}$ in $2.5^{\circ}$ increments, with a 300 s equilibration time at each temperature before acquiring ${ }^{1} \mathrm{H}$ (400.13 MHz) NMR spectra. All spectra were referenced to the residual $\mathrm{H}_{2} \mathrm{O}$ signal at $4.7 \mathrm{ppm}$. Cloud point $\left(T_{\mathrm{CP}}\right)$ measurements were performed by heating a solution of the polymer in water, deuterated water $\left(\mathrm{D}_{2} \mathrm{O}\right)$ or buffer at different concentrations (1.0, $2.5,5.0,10.0 \mathrm{mg} \mathrm{mL}^{-1}$ ) from $15-100{ }^{\circ} \mathrm{C}$, with a heating/cooling rate of $1.0^{\circ} \mathrm{C} \mathrm{min}^{-1}$, and $1 \mathrm{~s}$ equilibration time. Two heating/ cooling cycles were conducted, with the reported $T_{\mathrm{CP}}$ values calculated from absorbance measurements obtained in the first cycle. Absorbance $(A)$ values were converted to percent transmittance (\% $T$ ) by using this equation: $\% T=100\left(10^{-A}\right)$. Absorbance of the solutions during these cycles was recorded using a Shimadzu UV-1650 UV/Vis spectrometer with an 8-micro multicell temperature controlled cuvette chamber (path length 10 $\mathrm{mm}$ ), operated using a Shimadzu temperature controller and $T_{\mathrm{m}}$ analysis software. Reported $T_{\mathrm{CP}}$ values correspond to the temperature at half $(50 \%)$ of the transmittance variation. Buffer solutions used were as follows: $\mathrm{pH}$ 3: citric acid-sodium phosphate dibasic, pH adjusted with orthophosphoric acid; $\mathrm{pH}$ 7: sodium phosphatesodium phosphate dibasic, $\mathrm{pH}$ adjusted with orthophosphoric acid; $\mathrm{pH}$ 10.8: sodium bicarbonate-sodium carbonate buffer, $\mathrm{pH}$ adjusted with 1.0 $\mathrm{M} \mathrm{NaOH}$. The salt solutions used were either sodium bicarbonate $\left(\mathrm{NaHCO}_{3}\right)$ or sodium chloride $(\mathrm{NaCl})$ which was dissolved in Milli-Q grade water at a concentration of either $0.01,0.1,0.2,0.3,0.4,0.5,0.6,0.7,0.8,0.9$, or $1.0 \mathrm{M}$.

\section{General procedure for the preparation of statistical copolymers via RAFT}

Weighed quantities of monomers (DMAPMAm, MAA and tBMAm), RAFT agent (CETCPA), initiator (ACVA) and the 
internal standard (1,3,5-trioxane) were combined into $25 \mathrm{~mL}$ screw-top vials and diluted with solvent (either Milli-Q grade water, 1,4-dioxane, or glacial acetic acid) to a set concentration (usually $2.0 \mathrm{M}$ ). Each mixture was agitated to solubilise all components and a small aliquot was taken from each vial for NMR analysis ( $t=0$ time point). A rubber septum was then fitted to the top of each vial and oxygen was removed via a nitrogen purge for $30 \mathrm{~min}$. The vials were then placed in a temperature controlled oil bath that had been pre-heated to $70{ }^{\circ} \mathrm{C}$. Aliquots were taken at various time points and analysed by NMR to follow the course of the polymerisations. At the conclusion of the reactions, each vial was cooled to room temperature and opened to oxygen to quench the polymerisation. The resultant polymers were isolated by dialysis (SpectraPor6 RC tubing, MWCO $3.5 \mathrm{kDa}$ ) and were dialysed against Milli-Q grade water. Water was replaced 6 times over the course of the dialysis. Cleaned samples were then transferred to round bottomed flasks, frozen via immersion in liquid nitrogen and then dried using a freeze dryer. All polymers were isolated as fluffy white solids. Monomer consumptions, overall polymer conversions and the ratio of monomers in the final polymers were calculated from ${ }^{1} \mathrm{H}$ NMR spectra by comparison of the integrals of the vinylic protons at the beginning and the end of polymerisations against the integral of the internal standard at $\sim 5.2$ ppm. Full experimental details of all polymerisations can be found in the ESI section. $\dagger$

\section{Results and discussion}

\section{Polymer synthesis}

Using a combination of $\mathrm{N}$-[3-(dimethylamino)propyl]methacrylamide (DMAPMAm) and methacrylic acid (MAA) as the basic and acidic comonomers, respectively, we report a set of polyampholytes that have increasing amounts of $\mathrm{N}$-(tert-butyl) methacrylamide (tBMAm) incorporated as the hydrophobic comonomer. All polymers were synthesised from a combination of DMAPMAm, MAA and tBMAm monomers, via RAFT polymerisation ${ }^{34}$ using 4-((((2-carboxyethyl)thio) carbonothioyl)thio)4-cyanopentanoic acid (CETCPA) as the RAFT agent, as shown in Scheme 1.

In all cases the proportion of DMAPMAm to MAA was kept constant at a $1: 1$ ratio, and the content of tBMAm was varied from $0-50 \%$ (of total monomers), as shown in Table 1 . The purpose of this was to try and incorporate equimolar amounts of charged monomers, whilst also reducing the overall hydrophilicity of the terpolymers through incorporation of the hydrophobic monomer tBMAm. We hypothesised that this would help modulate the cloud points of the final polymers in solution. The purpose of synthesising the polymers via RAFT polymerisations was to ensure that the polymer chain lengths were controlled, minimising any interference from chain lengths arising from differences in polymer chain composition.

In determining the optimal conditions for the synthesis of these polymers, a variety of solvents were trialled (Table 1). Both DMF and absolute ethanol were initially trialled to synthesise 0$15 \%$ tBMAm copolymers (data not shown), however the contents of many of the reaction vials contained solid precipitates after only 1-2 hours of polymerisation, and low conversions were obtained as observed via NMR analysis. These same problems were also encountered when 1,4-dioxane was used (Table 1, forming polymers D1-D4), but were alleviated when Milli-Q grade water (Table 1, forming polymers W1-W4) or glacial acetic acid (Table 1, forming polymers A1-A8) were used. In the cases of both water and glacial acetic acid, all reaction components (and products) remained solubilised throughout the course of the polymerisation, which led to much higher monomer conversions. Following quenching of the polymerisation process, the polymers were purified using dialysis against Milli-Q grade water, and were freeze-dried to yield white fluffy solids. They were then analysed by ${ }^{1} \mathrm{H}$ NMR and GPC, with the results shown in Table 1.

Total monomer conversions, molecular weights $\left(M_{\mathrm{n}, \mathrm{NMR}}\right)$, the degree of polymerisation ( $\left.\mathrm{DP}_{\mathrm{NMR}}\right)$ and the percent monomer incorporation ( $\% \mathrm{M}_{1}: \mathrm{M}_{2}: \mathrm{M}_{3}$ ) were all determined from ${ }^{1} \mathrm{H}$ NMR analysis of samples taken at relevant time points over the course of the polymerisation. By analysing the integrals of peaks corresponding to the vinylic moieties of the monomers against the peak corresponding to the internal standard 1,3,5-trioxane, monomer conversions and the relative ratio of monomers incorporated into each polymer was calculated.

GPC analysis of all polymers was also conducted, and compared with the data obtained from NMR. There was some agreement between the experimentally derived molecular weights determined from different experimental methodologies $\left(M_{\mathrm{n}, \mathrm{GPC}}\right.$ vs. $M_{\mathrm{n}, \mathrm{NMR}}$; Table 1$)$, however in many cases large differences were observed due to differences in solvent $\left(\mathrm{H}_{2} \mathrm{O} v s\right.$. $\mathrm{D}_{2} \mathrm{O} v s$. pH 8 buffer; see Experimental section for further details) and structural differences to the PEO used for calibration of the

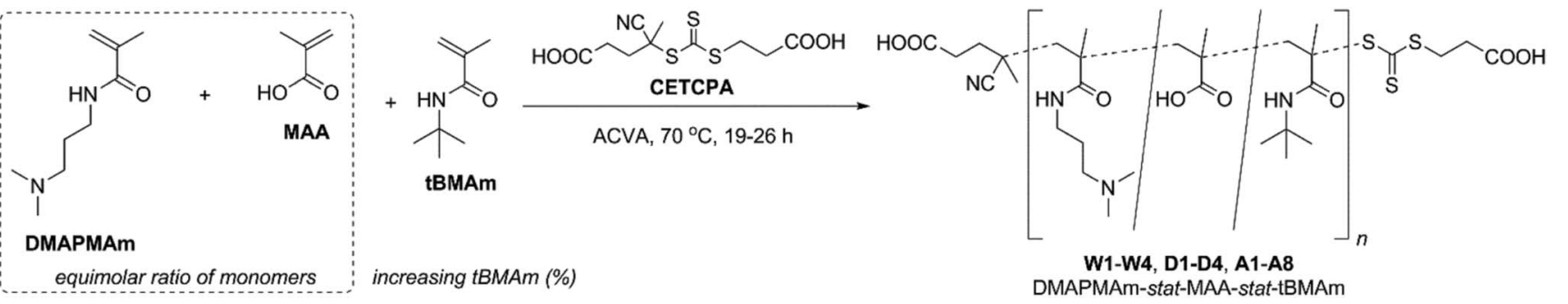

Scheme 1 Synthesis of DMAPMAm-stat-MAA-stat-tBMAm copolymers W1-W4, D1-D4 and A1-A8 via RAFT polymerisation, using CETCPA, $A C V A$, and a range of different solvents $(W=$ water; $D=1,4$-dioxane; $A=$ glacial acetic acid). 
Table 1 Experimental parameters used during the synthesis of DMAPMAm-stat-MAA-stat-tBMAm copolymers W1-W4, D1-D4 and A1-A8, and the results of polymer analysis obtained by ${ }^{1} \mathrm{H}$ NMR and GPC

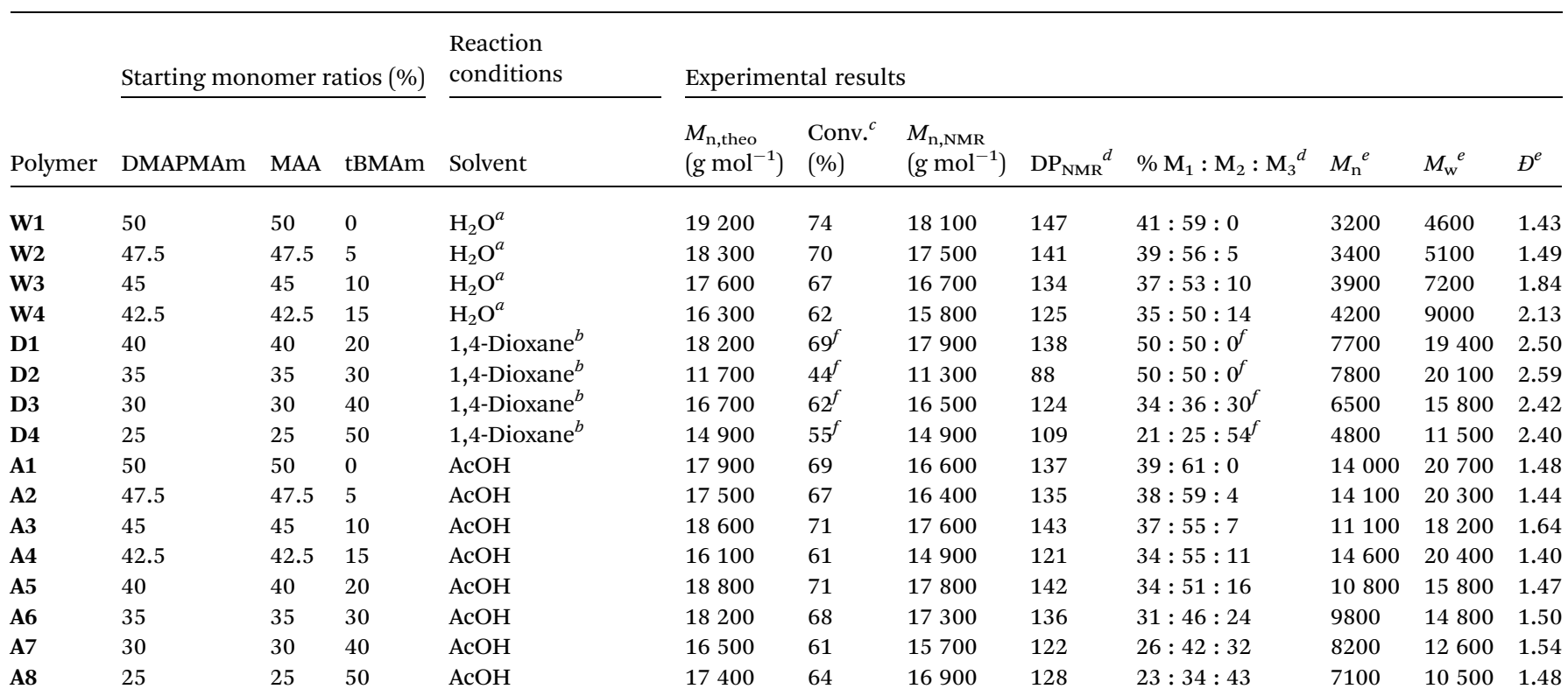

${ }^{a}$ Trace amounts of EtOH was needed to solubilise some starting materials. ${ }^{b}$ Precipitate formed after $1-2$ h. ${ }^{c}$ Percent (\%) overall conversion of monomer to polymer as calculated from ${ }^{1} \mathrm{H}$ NMR. ${ }^{d}$ Calculated from the integrals of the vinylic peaks against the integral of the internal standard 1,3,5-trioxane. ${ }^{e}$ Calculated from GPC (aqueous) against polyethylene glycol/oxide standards. ${ }^{f}$ Could not be calculated accurately due to insolubility of components of the reaction mixture in 1,4-dioxane and deuterated ${ }^{1} \mathrm{H}$ NMR solvents.

GPC. As the proportion of tBMAm relative to DMAPMAm and MAA is increased in the polymers however, these values become increasingly more disparate (A6-A8 in particular). This is most likely due to the increasing hydrophobicity of the polymer caused by altered interactions of the polymer side chains with the buffer solution at the $30{ }^{\circ} \mathrm{C}$ temperature used for GPC analysis.

GPC analysis showed the molar mass distributions to be in the range $D=1.40-2.59$. For all polymers synthesised in acetic acid (A1-A8) the dispersity values were the lowest $(\nexists=1.40-$ 1.64, Fig. 1c) compared with polymers synthesised in water $(\nexists=$ 1.43-2.13; Fig. 1a) or 1,4-dioxane $(\nexists=2.40-2.59$; Fig. $1 \mathrm{~b})$. Despite using RAFT polymerisation conditions to synthesise these polymers, there was clearly a loss of livingness during the synthesis of all of these polymers as shown by the high polydispersity values. In order to obtain a set of polymers that had similar molecular weights, we extended the polymerisation times up to 26 hours, which must have led to increased termination and side reactions occurring. Furthermore, for polymers W1-W4, hydrolysis of the RAFT group is known to occur under prolonged water exposure during polymerisation and is the most likely reason for presence of the higher molecular weight tailing (Fig. 1a). In the case of 1,4-dioxane, the higher molecular weight shoulders presumably arose from the insolubility of the products in the solvent which was evidenced by precipitation of the products from the reaction after only 2 hours of heating. This would have caused a range of polymer chain lengths to be present at the conclusion of the reaction, and is reflected in the
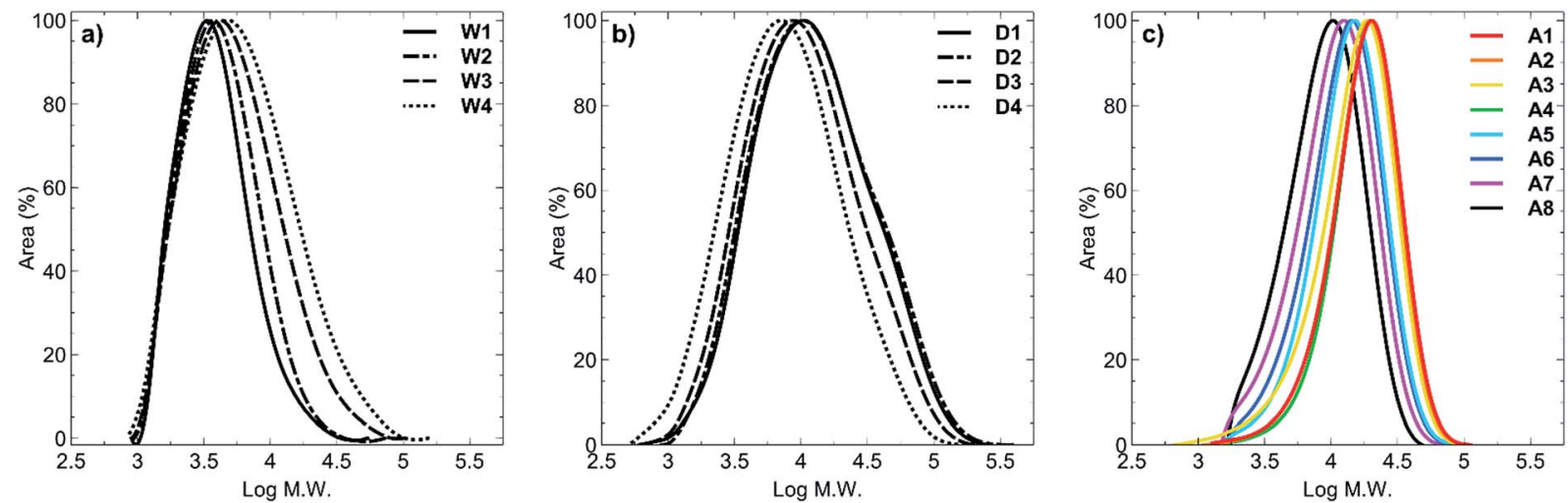

Fig. 1 GPC traces of polymer sets (a) W1-W4, (b) D1-D4, and (c) A1-A8. Solvent: Milli-Q grade water containing 200 mM sodium nitrate, 10 mM sodium phosphate and $200 \mathrm{ppm}$ sodium azide at $\mathrm{pH}$ 8; $M_{\mathrm{n}}$ are relative to polyethylene glycol/oxide (PEG/PEO) standards. 
broad peaks obtained (Fig. 1b). Polymers A1-A8 (Fig. 1c) clearly display a narrower dispersity range, compared to polymers W1W4 (Fig. 1a) and polymers D1-D4 (Fig. 1b), with minimal to no tailing effects observed in any of the acetic acid-synthesised polymers. This likely resulted from synthesis of these polymers under acidic conditions whereby hydrolysis of the RAFT end group was minimised.

The discrepancies in the above results are a clear indicator that the choice of solvent was crucial during the synthesis of these polymers, as previously highlighted. Solvent choice also impacted slightly on the monomer incorporation, particularly in the case of polymers W1-W4 and A1-A4. This was determined by ${ }^{1} \mathrm{H}$ NMR analysis of aliquots of reaction solutions collected during the reaction. From this data, the mole fraction of each monomer at a particular time point was determined, and plotted against overall monomer conversion. One example is shown in Fig. 2 for polymers with a starting feed of $42.5: 42.5: 15$ (DMAPMAm : MAA : tBMAm) synthesised in water (W4) versus the same polymer feed but synthesised in glacial acetic acid (A4). For a comparison of the remaining polymers, see Fig. S9-S15 in the ESI section. $\dagger$ Despite beginning with an initial $1: 1$ ratio of DMAPMAm to MAA, there is clearly greater incorporation of MAA into the polymer chain in both cases, as shown by the decrease in the mole fraction of MAA remaining over the course of the polymerisation (signifying its incorporation into the polymer chain). This is likely due to the different reactivity ratios of the two monomers, which have been reported as MAA $0.41 \pm 0.08$ and DMAPMAm $1.88 \pm 0.15$ for synthesis in water, ${ }^{35}$ compounded by the high selfassociation that has been reported for DMAPMAm. ${ }^{30}$ Whilst reactivity of this monomer pair might be expected to be vastly different in acetic acid rather than water, the incorporation of

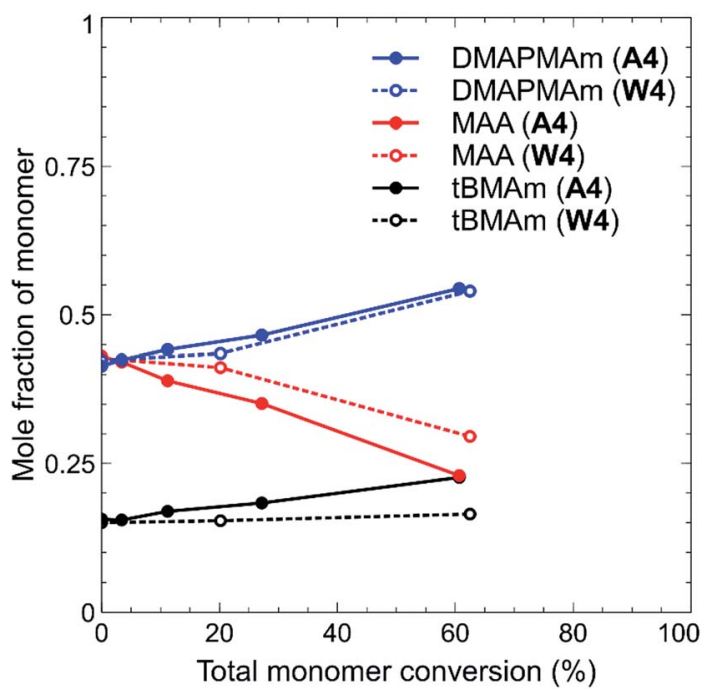

Fig. 2 Mole fraction of monomer as a function of total monomer conversion (\%) for polymers A4 and W4, as determined by ${ }^{1} \mathrm{H}$ NMR analysis of reaction aliquots. Data points and lines are coloured according to each monomer: blue - DMAPMAm; red - MAA; black tBMAm and are connected via solid lines (acetic acid-synthesised polymers) or dotted lines (water-synthesised polymers).
DMAPMAm does not significantly increase through this solvent change. There is, however, a much greater incorporation of tBMAm into the polymer chain when water is the solvent rather than acetic acid. Given that our research interests were focussed on observing and analysing the solution properties of these polymers, we did not estimate the reactivity ratio of these monomers during this study.

In order to best conserve the starting monomer composition, water would appear at this point to be the optimal solvent choice for the synthesis of these polymers. However, as shown by the GPC data, the polymers that were obtained from synthesis in water were much smaller than theoretical values, and their overall monomer composition and broader distribution of polymer chain molecular weights was much more variable, as demonstrated by their relatively high $D$ values. Therefore, the most reproducible and interesting polymers were obtained through synthesis in glacial acetic acid solvent. As such, only the stimuli-responsive properties of polymers A1-A8 will be discussed in the following sections.

\section{Temperature-responsive properties of polymers A1-A8}

The temperature-responsive properties of polymers A1-A8 at concentrations of $1.0,2.5,5.0$ and $10.0 \mathrm{mg} \mathrm{mL}^{-1}$ in Milli-Q grade water were determined via UV-Vis spectroscopy (500 $\mathrm{nm}$ ) at a heating/cooling rate of $1.0{ }^{\circ} \mathrm{C} \mathrm{min}^{-1}$ over the temperature range of $15-100{ }^{\circ} \mathrm{C}$. For each sample, the absorbance was measured and converted to the recommended percent transmittance (\% $\%$ ) values, ${ }^{19}$ and the cloud point temperature $\left(T_{\mathrm{CP}}\right)$ values were determined as described in the Experimental section.

The heating curves for polymers A1-A8 $\left(1.0 \mathrm{mg} \mathrm{mL} \mathrm{mL}^{-1}\right)$ are shown in Fig. 3, with the corresponding curves for the remaining concentrations $\left(2.5,5.0\right.$ and $\left.10.0 \mathrm{mg} \mathrm{mL}^{-1}\right)$ shown in the ESI section (Fig. S16(a)-(c) $\dagger$ ). In all cases, the polymer sets are ordered in increasing percent of tBMAm content, which ranges from $0 \%-43 \% \mathbf{A 1 - A 8}$, respectively. This is shown in each graph through different data point colours and sizes, where the increasing size of the data point directly corresponds to the increasing tBMAm content in each polymer.

In Fig. 3, it can clearly be seen that the phase transition of polymers A1-A4, which have a tBMAm content of $11 \%$ or lower, are not very sharp nor does the solution become significantly cloudy (solution transmittance is $>85 \%$ ). When the tBMAm content is $16 \%$ or higher however (A5-A8), the cloud point curves of the polymers become much sharper and the reduction in transmittance is much greater. This particular trend is graphically represented in Fig. 4, where polymers with a clearly defined $T_{\mathrm{CP}}$ value (A5-A8) have been plotted against tBMAm content (\%). By fitting a second order polynomial curve to this data, a very good correlation is observed between the cloud point temperatures and the hydrophobic content of the polymer chains. This analysis could potentially be used as a predictor of $T_{\mathrm{CP}}$ based on tBMAm incorporation in this system, assuming the relative molar ratio and incorporation of DMAPMAm and MAA monomers into each polymer is kept constant. Similar 


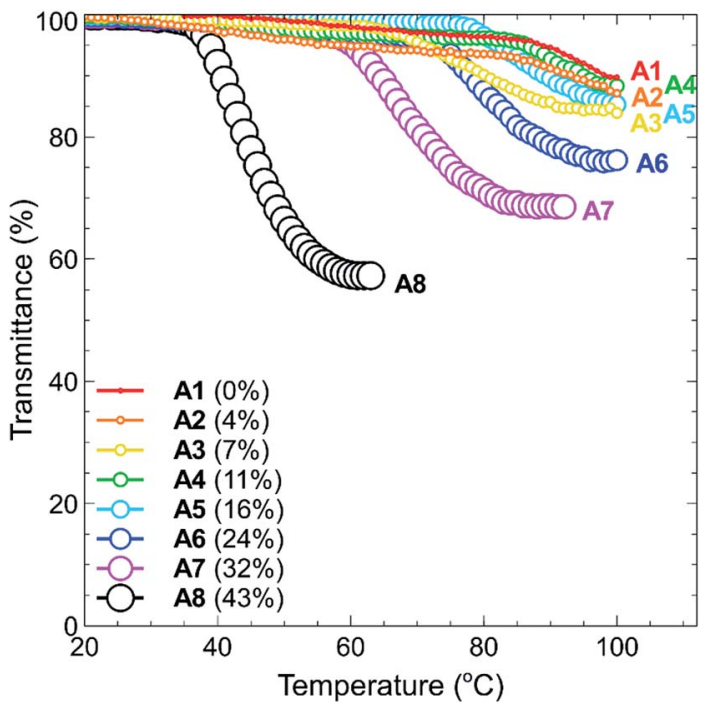

Fig. 3 Temperature $\left({ }^{\circ} \mathrm{C}\right.$ ) dependence of the transmittance (\%) of $1 \mathrm{mg}$ $\mathrm{mL}^{-1}$ solutions of polymers A1-A8 in Milli-Q grade water. Data point sizes and colours are set according to the relevant incorporation of tBMAm into each polymer, with the calculated percent of tBMAm in each polymer listed in brackets beside the polymer code.

results with good correlations are also observed at higher polymer concentrations, as shown in the ESI (Fig. S17(a)-(c)†).

Polymer concentration effects on the cloud points were also studied, with $T_{\mathrm{CP}}$ as a function of increasing polymer concentration plotted in Fig. 5(a) and (b). In all cases, the cloud point of each polymer decreased as the concentration was increased. This effect is caused by decreased solvation of the polymer in solution and is a commonly reported phenomenon for many different thermoresponsive polymers. ${ }^{19,36}$ What is most interesting from these results is that at a polymer concentration of $10 \mathrm{mg} \mathrm{mL}{ }^{-1}$, the polymers containing little to no tBMAm content have similar cloud points to those containing a high percentage of tBMAm, despite them having very different cloud points at much lower polymer concentrations. For example, the $T_{\mathrm{CP}}$ of $\mathbf{A 1}(0 \% \mathrm{tBMAm})$ at $10 \mathrm{mg} \mathrm{mL}^{-1}$ is $39^{\circ} \mathrm{C}$, whilst the $T_{\mathrm{CP}}$ of

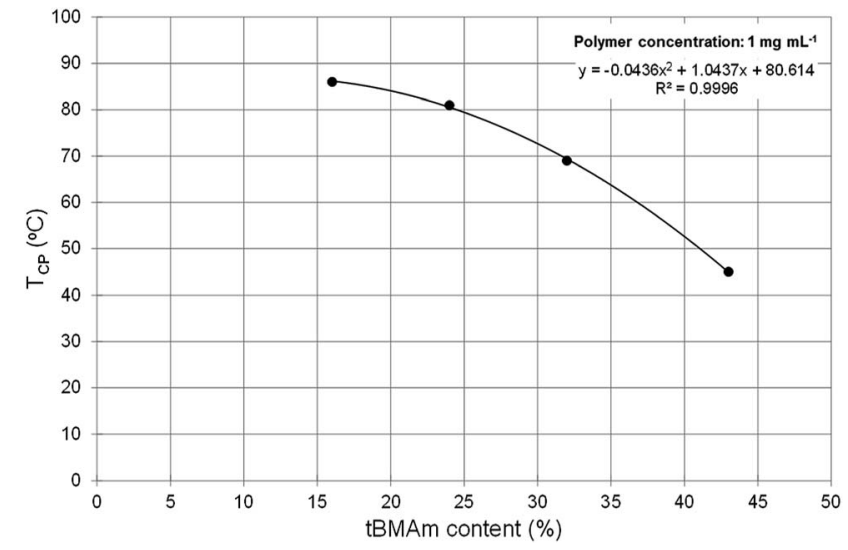

Fig. 4 Cloud point temperatures $\left(T_{\mathrm{CP}},{ }^{\circ} \mathrm{C}\right)$ vs. molar ratio of tBMAm content (\%) in polymers $\mathrm{A} 5-\mathrm{A} 8$ at $1 \mathrm{mg} \mathrm{mL}^{-1}$. A clear correlation is observed between decreasing $T_{\mathrm{CP}}$ and increasing tBMAm content.
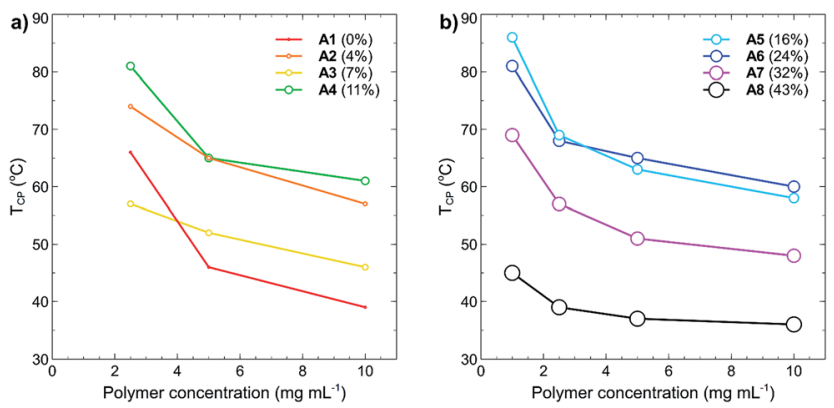

Fig. 5 Cloud points $\left(T_{\mathrm{CP}},{ }^{\circ} \mathrm{C}\right)$ of polymers (a) A1-A4 and (b) A5-A8 as function of polymer concentration $\left(\mathrm{mg} \mathrm{mL}^{-1}\right)$ in Milli-Q grade water. No $T_{C P}$ was recorded for polymers A1-A4 at $1 \mathrm{mg} \mathrm{mL}^{-1}$ (see Fig. 3). Data points are coloured and sized according to the increasing percent of tBMAm in each polymer.

A8 (43\% tBMAm) at the same polymer concentration is $36{ }^{\circ} \mathrm{C}(\mathrm{a}$ difference of $\left.3{ }^{\circ} \mathrm{C}\right)$. At $1 \mathrm{mg} \mathrm{mL}^{-1}$ however, polymer $\mathbf{A 1}(0 \%$ tBMAm) doesn't display a measureable cloud point, whereas the cloud point for polymer $\mathbf{A 8}\left(43 \%\right.$ tBMAm) is $45{ }^{\circ} \mathrm{C}$; a difference of over $55{ }^{\circ} \mathrm{C}$. These effects likely result from the increased selfassociation of polymers under these high concentrations, which leads to a decrease in hydrogen bonding capacity of the polymers with water and overall lowered $T_{\mathrm{CP}}$.

\section{pH and salt effects on the polymer cloud points}

By incorporating a balance of acidic and basic comonomers into the polymer chains, it was expected that these polymers would display additional responsiveness to changes in $\mathrm{pH}$. To test this hypothesis, each polymer A1-A8 was dissolved at a concentration of $1 \mathrm{mg} \mathrm{mL}^{-1}$ in $10 \mathrm{mM}$ buffer solutions that had been adjusted to $\mathrm{pH} 3,7$, and 10 (see Experimental section for buffer details). These values were selected as the $\mathrm{pH}$ extremities 3 and 10 were greater than the $\mathrm{p} K_{\mathrm{a}}$ 's of each of the monomers in the chain, namely, 4.66 for $\mathrm{MAA}^{37}$ and 9.25 for DMAPMAm. ${ }^{38}$ The $\mathrm{pH}$ values of the polymer solutions were then measured using a $\mathrm{pH}$ probe to ensure that the final solution $\mathrm{pH}$ matched that of the buffer solution. In all cases no change in $\mathrm{pH}$ was observed from the measured $\mathrm{pH}$ of the buffer solution. Each polymer-buffer solution was then heated and analysed via turbidimetry (as previously outlined in the Experimental section) to determine the polymer cloud points. In all cases however, no decrease in transmittance was observed for any polymer solutions at any of the three pH's tested. This is most likely an effect of the polyzwitterionic nature of these polymers, which act as buffers themselves against changes in $\mathrm{pH}$. At $\mathrm{pH} 3$ and 10, the acid and base groups of the polymers were each neutralised, respectively, and so the polymers each contained a single charge at each of these $\mathrm{pH}$ 's. This would have led to an increase in the solubility of the polymers in water akin to the behaviour of typical polyelectrolyte thermoresponsive polymers.

The thermoresponsive properties of the polymers also changed from the addition of salt. Each of the polymers was dissolved at a concentration of $1 \mathrm{mg} \mathrm{mL}{ }^{-1}$ in either $\mathrm{NaHCO}_{3}$ or $\mathrm{NaCl}$ solutions at salt concentrations ranging between 0.01- 


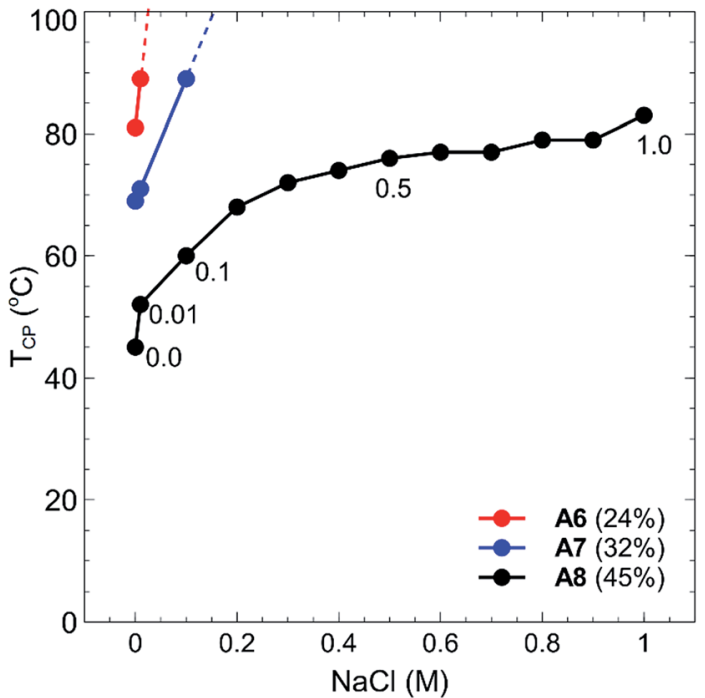

Fig. 6 Cloud point temperatures $\left(T_{\mathrm{CP}},{ }^{\circ} \mathrm{C}\right)$ vs. sodium chloride concentration ( $\mathrm{NaCl}, \mathrm{M}$ ) for polymers $\mathrm{A} 6$ (red), A7 (blue) and A8 (black) at $1 \mathrm{mg} \mathrm{mL}^{-1}$ in Milli-Q grade water, with the tBMAm (\%) in each polymer shown in brackets.

1.0 M. Again, the cloud points for all polymers increased drastically, with polymers A1-A5 displaying no $T_{\mathrm{CP}}$ events at any of the salt concentrations tested. In the case of polymers A6-A8, no cloud points were observed in $\mathrm{NaHCO}_{3}$ salt solutions, whilst some $T_{\mathrm{CP}}$ events were observed in $\mathrm{NaCl}$ solutions as shown in Fig. 6. Polymers A6, $\mathbf{A 7}$ and $\mathbf{A 8}$ all showed marked increases in their cloud points as salt was added, particularly when the concentration was increased from $0.01-0.1 \mathrm{M}$, with no cloud points observed for polymers $\mathbf{A 6}$ and $\mathbf{A 7}$ above this range. Whilst the cloud point of polymer $\mathbf{A 8}$ showed a steep increase in temperature at low salt concentrations, this increase plateaued off somewhat as the salt concentration was further increased up to $1.0 \mathrm{M}$.

As previously mentioned, polyelectrolytes and other noncharged thermoresponsive polymers generally display a decrease in their $T_{\mathrm{CP}}$ as the salt concentration is increased. This is due to the fact that the addition of ions shields the polymer from water, which creates a more hydrophobic environment for the polymer and results in a decreased $T_{\mathrm{CP}}$ value. Due to the presence of both anionic and cationic charged moieties in these polymers however, properties akin to traditional polyzwitterions are displayed, wherein polymer solubility is actually increased as a function of salt concentration due to the shielding of the electrostatic interactions of the polyampholytes. ${ }^{39}$

\section{NMR studies of polymer A8}

To examine these effects in more detail, NMR was employed to assess the structure of polymer $\mathbf{A 8}$ in the presence and absence of salt at temperatures above and below the determined cloud points. NMR is an excellent tool to study the thermoresponsive properties of polymers at the molecular level because decreased chain mobility in solution and the relative hydration states of specific moieties in a polymer chain can be visualised through broadening of polymer peaks above the polymer cloud point. ${ }^{19}$ In this study, $10 \mathrm{mg} \mathrm{mL}{ }^{-1}$ solutions of polymer $\mathbf{A 8}$ both in the presence $(0.01 \mathrm{M} \mathrm{NaCl})$ and absence $(0.0 \mathrm{M})$ of salt were heated in situ at $2.5{ }^{\circ} \mathrm{C}$ increments from $25{ }^{\circ} \mathrm{C}$ to $75^{\circ} \mathrm{C}(300 \mathrm{~s}$ equilibration time at each temperature), with ${ }^{1} \mathrm{H}$ NMR spectra acquired at the various temperature intervals. The arrayed NMR spectra of the temperature intervals $\left(5{ }^{\circ} \mathrm{C}\right.$ for simplicity) were then plotted and are shown in the ESI section (Fig. S18: $\uparrow \mathrm{A8}$ in $\mathrm{D}_{2} \mathrm{O}(0 \mathrm{mM} \mathrm{NaCl})$ and Fig. $\mathrm{S} 19: \dagger \mathbf{A 8}$ in $\left.\mathrm{D}_{2} \mathrm{O}(10 \mathrm{mM} \mathrm{NaCl})\right)$. In both cases, all peaks exhibit broadening and downfield shifts as the temperature of the solution is increased. This indicates that there is loss of water surrounding the polymers as they transition from a hydrophilic state to a hydrophobic state. Furthermore, there is no observable difference here between the shape and/or broadening of each spectra from the addition of salt. This shows that salt does not appreciably affect the side chains in this polymer that might otherwise cause differences in hydration, which would be observed by sharpening or
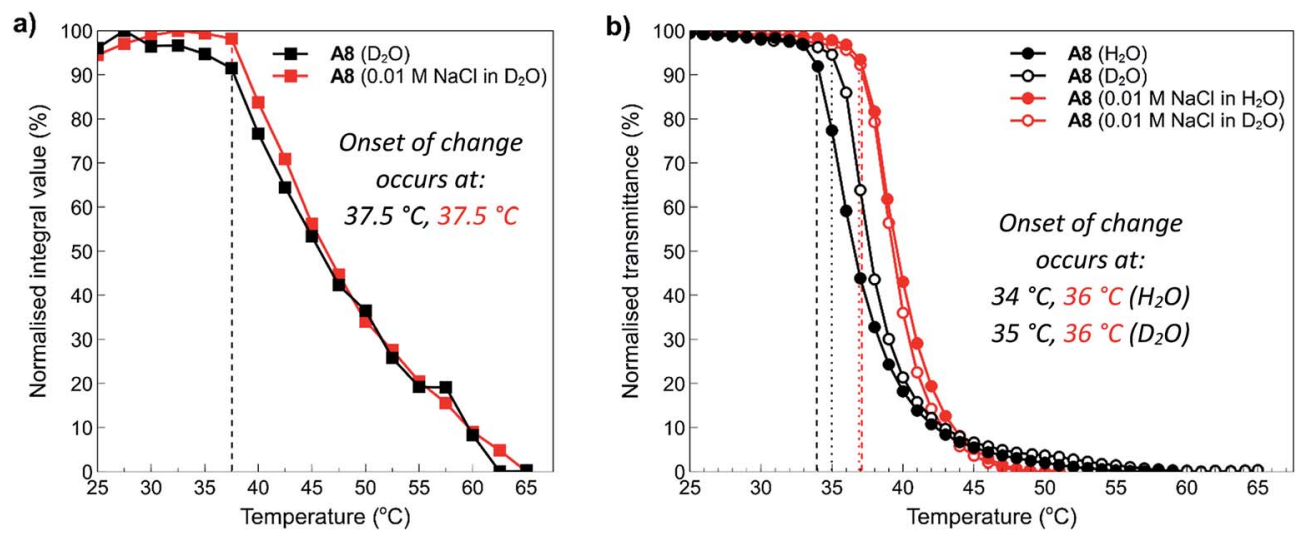

Fig. 7 (a) Temperature dependence of the normalised integral of peaks corresponding to polymer side chain and backbone protons as measured by in situ temperature ramp ${ }^{1} \mathrm{H}$ NMR experiments in $\mathrm{D}_{2} \mathrm{O}$ (black squares) and $10 \mathrm{mM} \mathrm{NaCl}$ in $\mathrm{D}_{2} \mathrm{O}$ (red squares). (b) Temperature dependence of the transmittance (\%) of $10 \mathrm{mg} \mathrm{mL}^{-1}$ solutions of polymer $\mathrm{A} 8$ in Milli-Q grade water (filled black circles), $\mathrm{D}_{2} \mathrm{O}$ (open black circles), $0.01 \mathrm{M} \mathrm{NaCl}$ in Milli-Q grade water (filled red circles) and $0.01 \mathrm{M} \mathrm{NaCl}$ in $\mathrm{D}_{2} \mathrm{O}$ (open red circles). 
broadening of specific peaks corresponding to the polymer pendant groups.

Data from these experiments was then collated to obtain further information about the temperature dependence of the polymer solubilities. Normalised integrals of the region between 0.5-4.0 ppm corresponding to peaks representing the side chain and backbone protons of the polymers were compared to the integral of residual $\mathrm{H}_{2} \mathrm{O}$ in each sample, and this data was plotted against temperature as shown in Fig. 7a. From this graph, it can clearly be seen that after $37.5{ }^{\circ} \mathrm{C}$, the polymers start to aggregate and phase separate from the solution. Furthermore, there is no appreciable difference in the temperature at which this occurs despite the addition of salt. This can also be observed in the spectra (see ESI, Fig. S18 and $\mathrm{S} 19 \dagger)$ where a decrease in the intensity of the peaks is noticeable as the temperature is increased. Shown alongside the NMR data in Fig. 7a is the equivalent data obtained for this polymer via turbidimetry (Fig. 7b). In this case, the onset of change in the polymer system occurs at slightly lower temperatures; $34{ }^{\circ} \mathrm{C}$ and $35{ }^{\circ} \mathrm{C}$ for the solution in pure water and $\mathrm{D}_{2} \mathrm{O}$, respectively, and $36{ }^{\circ} \mathrm{C}$ for the $0.01 \mathrm{M} \mathrm{NaCl}$ solutions. This data is roughly in agreement with that observed during the temperature ramp experiments, with small changes most likely resulting from a combination of experimental error and slight differences in hydrogen vs. deuterium bonding interactions with the polymers. This data further demonstrates the polyzwitterionic properties of the polymers, where at high polymer concentrations they are able to accommodate small changes in salt concentrations.

\section{Conclusion}

A series of $N$-[3-(dimethylamino)propyl]methacrylamide (DMAPMAm), methacrylic acid (MAA) and $\mathrm{N}$-(tert-butyl)methacrylamide (tBMAm) terpolymers were synthesised via RAFT polymerisation. Polymer synthesis in a variety of solvents was trialled, and glacial acetic acid was determined to be the best solvent during this synthesis as the polymers obtained had a narrow molecular weight distribution that was close to theoretical values. Polymers A1-A8 with a range of tBMAm contents were then characterised for their response to changes in temperature, $\mathrm{pH}$ and salt concentrations. At all polymer concentrations ranging between $1-10 \mathrm{mg} \mathrm{mL}^{-1}$, polymer cloud points were successfully modulated by the inclusion of tBMAm, with the cloud points reduced as the tBMAm content was increased to $16 \%$ and above. Changes in $\mathrm{pH}$ and addition of salt both led to an increase in the polymer cloud points, and in many cases, rendered the polymers free of their thermoresponsive properties within the $15-100{ }^{\circ} \mathrm{C}$ temperature ranges investigated.

\section{Acknowledgements}

The authors wish to acknowledge funding from the CSIRO's Office of the Chief Executive (OCE). Many thanks to Dr Roger Mulder for his help with the NMR experiments and subsequent analyses, and to Dr David Alexander for his help with the data analysis of the cloud point temperatures versus molar ratio of tBMAm content.

\section{References}

1 M. L. Wei, Y. F. Gao, X. Li and M. J. Serpe, Polym. Chem., 2017, 8, 127-143.

2 M. Talelli, A. Duro-Castaño, G. Rodríguez-Escalona and M. J. Vicent, in Smart Polymers and their Applications, ed. M. R. Aguilar and J. S. Román, Woodhead Publishing, 2014, pp. 327-358, DOI: 10.1533/9780857097026.2.327.

3 C. A. Custódio, R. L. Reis, J. F. Mano and A. Del Campo, in Smart Polymers and their Applications, ed. M. R. Aguilar and J. S. Román, Woodhead Publishing, 2014, pp. 301-326, DOI: 10.1533/9780857097026.2.301.

4 M. A. Cohen Stuart, W. T. S. Huck, J. Genzer, M. Muller, C. Ober, M. Stamm, G. B. Sukhorukov, I. Szleifer, V. V. Tsukruk, M. Urban, F. Winnik, S. Zauscher, I. Luzinov and S. Minko, Nat. Mater., 2010, 9, 101-113.

5 P. J. Roth and A. B. Lowe, Polym. Chem., 2017, 8, 10-11.

6 A. S. Hoffman, Macromol. Symp., 1995, 98, 645-664.

7 G. Kocak, C. Tuncer and V. Bütün, Polym. Chem., 2017, 8, 144-176.

$8 \mathrm{~J}$. Thevenot, H. Oliveira, O. Sandre and S. Lecommandoux, Chem. Soc. Rev., 2013, 42, 7099-7116.

9 Z. Sekkat and W. Knoll, in Molecular Switches, Wiley-VCH Verlag GmbH \& Co. KGaA, 2011, pp. 423-515, DOI: 10.1002/9783527634408.ch13.

10 D. A. Davis, A. Hamilton, J. Yang, L. D. Cremar, D. Van Gough, S. L. Potisek, M. T. Ong, P. V. Braun, T. J. Martinez, S. R. White, J. S. Moore and N. R. Sottos, Nature, 2009, 459, 68-72.

11 R. Hoogenboom, in Smart Polymers and their Applications, ed. M. R. Aguilar and J. S. Román, Woodhead Publishing, 2014, pp. 15-44, DOI: 10.1533/9780857097026.1.15.

12 H. G. Schild, Prog. Polym. Sci., 1992, 17, 163-249.

13 J.-F. Lutz, Ö. Akdemir and A. Hoth, J. Am. Chem. Soc., 2006, 128, 13046-13047.

14 R. Pelton, J. Colloid Interface Sci., 2010, 348, 673-674.

15 J. F. Lutz, Adv. Mater., 2011, 23, 2237-2243.

16 G. Vancoillie, D. Frank and R. Hoogenboom, Prog. Polym. Sci., 2014, 39, 1074-1095.

17 M. Heskins and J. E. Guillet, J. Macromol. Sci., Part A: Pure Appl. Chem., 1968, 2, 1441-1455.

18 R. Hoogenboom, in Complex Macromolecular Architectures, John Wiley \& Sons (Asia) Pte Ltd, 2011, pp. 685-715, DOI: 10.1002/9780470825150.ch22.

19 Q. Zhang, C. Weber, U. S. Schubert and R. Hoogenboom, Mater. Horiz., 2017, 4, 109-116.

20 M. Constantin, M. Cristea, P. Ascenzi and G. Fundueanu, eXPRESS Polym. Lett., 2011, 5, 839-848.

21 R. Freitag and F. Garret-Flaudy, Langmuir, 2002, 18, 3434-3440. 22 A. Kumar, A. Srivastava, I. Y. Galaev and B. Mattiasson, Prog. Polym. Sci., 2007, 32, 1205-1237.

23 M. Liu, C. Taylor, B. Chong, L. Liu, A. Bilic, N. S. Terefe, R. Stockmann, S. Thang and K. de Silva, Eur. Polym. J., 2014, 56, 153-159. 
24 N. S. Terefe, O. Glagovskaia, K. De Silva and R. Stockmann, Food Bioprod. Process., 2014, 92, 208-225.

25 D. Roy, W. L. A. Brooks and B. S. Sumerlin, Chem. Soc. Rev., 2013, 42, 7214-7243.

26 M. B. McCool and E. Senogles, Eur. Polym. J., 1989, 25, 857860.

27 D. Gromadzki, P. Štěpánek and R. Makuška, Mater. Chem. Phys., 2013, 137, 709-715.

28 K. Vallee-Rehel, V. Langlois and P. Guerin, J. Environ. Polym. Degrad., 1998, 6, 175-186.

29 P. van de Wetering, N. J. Zuidam, M. J. van Steenbergen, O. A. G. J. van der Houwen, W. J. M. Underberg and W. E. Hennink, Macromolecules, 1998, 31, 8063-8068.

30 D. M. Kamorin, M. Rumyantsev, O. A. Kazantsev, A. P. Sivokhin and S. I. Kamorina, J. Appl. Polym. Sci., 2017, 134, 44412.

31 A. B. Lowe and C. L. McCormick, Chem. Rev., 2002, 102, 4177-4190.
32 M. J. Fevola, J. K. Bridges, M. G. Kellum, R. D. Hester and C. L. McCormick, J. Appl. Polym. Sci., 2004, 94, 24-39.

33 G. S. Georgiev, E. B. Kamenska, E. D. Vassileva, I. P. Kamenova, V. T. Georgieva, S. B. Iliev and I. A. Ivanov, Biomacromolecules, 2006, 7, 1329-1334.

34 G. Moad, Polym. Chem., 2017, 8, 177-219.

35 R. A. Navolokina, V. A. Sinsokov, L. I. Abramova and S. M. Danov, Izv. Vyssh. Uchebn. Zaved., Khim. Khim. Tekhnol., 1996, 39, 191-193.

36 L. G. Weaver, R. Stockmann, A. Postma and S. H. Thang, RSC $A d v .$, 2016, 6, 90923-90933.

37 I. A. Efimenko, P. V. Ankudinova, L. G. Kuz'mina, A. V. Churakov, N. A. Ivanova, L. I. Demina and O. S. Erofeeva, Russ. J. Inorg. Chem., 2015, 60, 848-860.

38 O. A. Kazantsev, K. V. Shirshin, S. A. Kazakov and S. M. Danov, Russ. J. Inorg. Chem., 1996, 66, 2014-2018.

39 J. Zhao, N. A. D. Burke and H. D. H. Stover, $R S C A d v$., 2016, 6, 41522-41531. 